\title{
Study of Translation Teaching of Traditional Chinese Medicine Based on Corpus
}

\author{
Xiaofang Wang ${ }^{1, a}$ and Cheng Liu $^{2, b^{*}}$ \\ School of Humanities, Jiangxi University of TCM, No. 1688 of Meiling Road, Wanli District, \\ Nanchang City, Jiangxi Province, China \\ School of Humanities, Jiangxi University of TCM, No. 1688 of Meiling Road, Wanli District, \\ Nanchang City, Jiangxi Province, China \\ a920711622@qq.com, bjxjzlc@163.com \\ * The Corresponding Author
}

Keywords: Corpus; Traditional Chinese medicine English; Translation teaching; Reform of college English teaching

\begin{abstract}
With the deepening of the reform of College English teaching, the construction of the English corpus of traditional Chinese medicine is particularly important. Based on the theory of corpus linguistics, this paper expounds the significance of the construction of TCM English corpus in TCM English teaching, and further puts forward a series of principles for building relevant database.
\end{abstract}

\section{Introduction}

With the continuous deepening of the trend of the internationalization of traditional Chinese Medicine culture, the demand for both Chinese and English proficient talents is relatively large. The cultivation of this kind of compound talents mainly depends on the training of College English Teaching for students in Chinese medicine colleges and universities. At this stage, the teaching of College English in China is in an important period of transformation, which refers to the transfer of English for General Purpose (short for EGP) to English for Special Purpose (short for ESP). In this transition period, how to train the complex talents in accordance with the development of the times has become an important subject in the study of English Teaching in Universities of traditional Chinese medicine.

TCM English is an important branch of ESP. It mainly refers to a unique expression system gradually formed in the process of TCM translation and communication. At present, as a new subject, traditional Chinese medicine has been set up in most Chinese Colleges of traditional Chinese medicine. However, the construction of its corpus is a new topic in the transition period of College English teaching, which needs to be explored and studied in depth.

\section{Corpus and Corpus of Traditional Chinese Medicine English}

A corpus is a language gathering collected in a scientific way for a certain purpose of research. A corpus can accurately and comprehensively represent the linguistic features of a certain language or one of its aspects. Through corpus approach, a large number of texts are easily processed so that people can accurately distinguish the linguistic features between different types of text. With the rapid development of computer technology, the development and application of the Internet provide rich resources and scientific support for the successful construction of the corpus. Therefore, in the modern sense, corpus is mainly a collection of language materials that are built, stored and used by the computer as a tool. Through the corpus, language materials are retrieved, analyzed and processed to learn the characteristics and rules of the language so as to facilitate the application and teaching of language.

In recent years, corpus linguistics has been developing rapidly. Under the premise of modern Internet technology, corpus's deep application has received much attention from researchers. The construction of the English corpus of traditional Chinese medicine is a typical example. As the 
quintessence of Chinese traditional culture, TCM culture can only be internationalized through international language such as English, so as to achieve the purpose of promoting Chinese culture. Therefore, the construction of the English corpus of traditional Chinese medicine has become an important way of the international communication of traditional Chinese medicine culture. In order to meet the needs of the research and practice of traditional Chinese medicine, the English corpus of traditional Chinese medicine (TCM) is a collection of Chinese English text libraries, which are processed and stored by computer. The corpus of TCM is not only a simple retrieval corpus based on the vocabulary of Chinese medicine, but also a retrieval of English translation of TCM words and an analysis and summary of Chinese texts and pragmatics by selecting TCM culture.

\section{The Significance of the Construction of TCM English Corpus}

The construction of TCM English corpora is an inevitable requirement for the spread of Chinese medicine culture internationally, and it is also an objective requirement for training compound talents who know both Chinese medicine and English. The establishment of TCM English corpus can provide abundant teaching resources, and its research results can be used to guide teaching, syllabus design, and textbook compilation, learning mode, assessment and examination.

Firstly, improve the quality of English Teaching in traditional Chinese medicine. The English corpus of traditional Chinese medicine can provide rich and colorful materials for the teaching of traditional Chinese medicine. In addition to make objective judgment for common language structure, difficult language points in teaching, TCM English Corpus can also design learning tasks, organize classroom activities and subsequent learning through the context, so as to improve the quality of English Teaching of Chinese medicine.

Secondly, the design of the syllabus and the compilation of the teaching materials can be promoted. Through the function of word frequency query in TCM English corpus, we can find out the corpus often used in TCM English, so as to guide the design of syllabus, enable students to clearly understand learning objectives and curriculum requirements, and also lay stress on the key points and grasp the central points in compiling textbooks.

Thirdly, it can be used to create an exploratory learning model of human-computer interaction. The establishment of English corpus of traditional Chinese medicine (TCM) can be taught through multimedia and network. The related knowledge of TCM English curriculum can be communicated and communicated outside the classroom through multimedia network technology, and heuristic and analytical learning is helpful for students to learn independently and improve their interest in learning English.

Lastly, it can improve the evaluation and assessment of students' learning results. The use of TCM English corpus can evaluate and assess the key points and difficulties, the completion of teaching objectives and the understanding of students, so as to further guide the English Teaching of TCM.

\section{Construction of English Corpus of Traditional Chinese Medicine}

The target should be compatible. The establishment of TCM English corpus should include all fields related to TCM to the maximum extent, and also include the interactive corpus of Chinese synchronous English translation. The establishment of the English corpus of traditional Chinese medicine plays an important role in the translation of traditional Chinese medicine and the teaching and research of traditional Chinese medicine.

The content should be authentic. This is the fundamental principle of the construction of the English corpus of traditional Chinese medicine. We can collect suitable corpus from traditional Chinese medicine literature and Internet, and choose the published and international language expressions to ensure the correctness and rationality of the corpus.

The definite corpus should be of representativeness. When constructing the corpus of TCM English, the corpus that should be included should cover the various aspects related to the purpose of building the library. In addition, it should also represent the characteristics of a language or a 
specific domain as much as possible when designing corpus.

The structure of corpus should be reasonable. This involves the vertical depth of the construction of the corpus. First, we can set up a collection of text in a certain field, pay attention to the scope of coverage, and rationally configure the proportion of corpus matching among different contents, so as to ensure that each part of the corpus occupies a certain proportion.

The sampling should be randomly selected. This ensures the quality of the corpus and the representativeness of the sample. In order to represent an infinitely large population, corpora should strictly follow the principle of sampling randomness, retrieve data based on a key word, sort the acquired corpus, and then join corpus, and avoid the subjective intervention of artificial selection.

The storage capacity should be dynamic. Although some classic translations of traditional Chinese medicine are relatively fixed, the language itself is developed dynamically. The corpus manager can collect and annotate the collected corpus before entering the library, and at the same time, it will connect and communicate with other corpora, so TCM corpus can be enriched and perfected continuously.

Methods should be standardized. All the selected language materials must be marked and arranged to facilitate the retrieval and use. We can use the standard marking method of combining disease name identification position with medical branch types with disease name number with suffix numbers, integrate different types of corpus, and use Chinese corpus of TCM correspondingly. In addition, it is possible to mark the background information of the selected corpus and the key words in order to meet the different needs of translation, teaching and scientific research.

\section{Conclusion}

It can be seen easily that the construction of the English corpus of traditional Chinese medicine needs a lot of manpower, material and financial input. In the transformation of College English teaching reform of our country, the successful construction of TCM English corpus are not only conducive to the standardization of English translation of traditional Chinese medicine, but also can meet the needs of Chinese medicine English teaching training talents, and promote the process of internationalization of traditional Chinese medicine culture dissemination.

\section{Acknowledgements}

Key Project of Teaching Reform of Jiangxi University of Traditional Chinese Medicine (No: 2016jzzd-1); Research of Education Science Planning Projects of $13^{\text {th }}$ Five-Year in Jiangxi Province (No: 17YB145); Research Project of Humanities and Social Science for Colleges and Universities in Jiangxi Province (No: YY162002).

\section{References}

[1] Wen Yongyi, Fan Xinrong. Construction of TCM English Corpus [J]. Journal of Shaanxi University of Chinese Medicine, 2003, 26 (5).

[2] He Anping. Corpus linguistics and English teaching [M]. Foreign Language Teaching and Research Press, 2006.

[3] Li Zhaoguo. Chinese medicine English: a new subject being formed [J]. Journal of Shanghai University of Traditional Chinese Medicine, 1999, (3). 